\title{
Why Disagreement May Not Matter (much) for Asset Prices
}

\author{
Paul Söderlind* \\ This version: 9 September 2008 (First draft: 21 April 2008) \\ Forthcoming in Finance Research Letters
}

\begin{abstract}
A simple consumption-based two-period model is used to study the (theoretical) effects of disagreement on asset prices. Analytical and numerical results show that individual uncertainty has a much larger effect on risk premia than disagreement if $(i)$ the risk aversion is reasonably high and (ii) individual uncertainty is not much smaller than disagreement. Evidence from survey data on beliefs about output growth suggests that the latter is more than satisfied.
\end{abstract}

Keywords: equity premium, riskfree rate, implied volatility, Survey of Professional Forecasters

JEL: C42, G12, E44.

\section{Introduction}

The effect of disagreement on asset prices is an important and contested topic. A growing literature explores the theoretical underpinnings for and empirical evidence of using disagreement as a new "risk factor."1

*University of St. Gallen and CEPR. Address: SBF, University of St. Gallen, Rosenbergstrasse 52, CH-9000 St. Gallen, Switzerland. E-mail: Paul.Soderlind@unisg.ch. I thank Michael Fisher, a co-editor and a referee for comments.

${ }^{1}$ For instance, Anderson, Ghysel, and Juergens (2005), Basak (2005), David (2008), Shefrin (2005) and Kurz and Motolese (2007) analyse the effects in general equilibrium settings, Lintner (1969), Gerber and Hens (2006) and Fama and French (2007) use a CAPM setting, while Hong and Stein (2007) provides a general discussion of the link between price and trade volume dynamics. 
This short and simple paper employs a two-period endowment model (previously used by Varian (1985) and Giordani and Söderlind (2005)) to argue that basic asset pricing theory suggests that disagreement is not particularly important-at least not as long as the risk aversion is reasonably high and disagreement is of the same order of magnitude as individual uncertainty. The latter is backed up by evidence from the Survey of Professional Forecasters, which (uniquely) has information about both individual uncertainty and disagreement.

The way disagreement affects asset pricing is that most investors get good deals on assets they (not others) believe will pay off. This leads to little diversification, that is, highly volatile consumption. Assets that are correlated with consumption therefore require substantial risk premia. However, with high risk aversion, investors diversify to mitigate the volatility so the effect of disagreement is muted.

While the existing literature (see, for instance, the references in footnote 1) already show some of these results, it typically does so in much more complicated models-and often only implicitly. The contribution of this paper is to highlight when and how disagreement matters for asset pricing. To do that, a very simple model is used-which generates easily interpretable results. It is shown that the equity premium is approximately $\gamma \sigma^{2}+\delta^{2}$, where $\gamma$ is the relative risk aversion, $\sigma^{2}$ is the output variance and $\delta^{2}$ is a measure of disagreement. It is also shown that the implied variance of a stock option is approximately equal to the equity premium divided by $\gamma$. These results are exact for logarithmic utility $(\gamma=1)$ and/or when all investors agree-and good approximations for higher values of the risk aversion (even if investors disagree substantially). The approximations only fail when risk aversion is substantially lower than unity at the same time as disagreement is large.

The outline of the paper is as follows: Section 2 presents the portfolio and consumption choice problem and discusses the main assumptions made to solve the model; Section 3 derives the asset pricing implications of the portfolio choice; Section 4 presents survey evidence on individual uncertainty and disagreement; and Section 5 concludes with a brief discussion. Technical details are found in two appendices. 


\section{Disagreement and Portfolio Choice}

In this simple model, disagreement and individual uncertainty are kept distinct, markets are complete, there are only two periods and all investors are identical except for their beliefs about future GDP growth.

The objective of investor $i$ is to maximise expected CRRA utility from consumption in periods $1\left(C_{i 1}\right)$ and $2\left(C_{i 2}\right)$. The latter is a random variable (function) of the state that will be realised in period 2. Let $f_{i}(s)$ be shorthand notation for investor $i$ 's probability density function (pdf) of the random state $s$. The objective is then to maximise

$$
\frac{C_{i 1}^{1-\gamma}}{1-\gamma}+\beta \int_{s} \frac{C_{i 2}(s)^{1-\gamma}}{1-\gamma} f_{i}(s) d s .
$$

(Clearly, the integral in the second term is the expected value of $C_{i 2}^{1-\gamma} /(1-\gamma)$.) The budget constraints are

$$
\begin{aligned}
& Y_{i 1}=C_{i 1}+\int_{s} p(s) B_{i}(s) d s, \text { and } \\
& C_{i 2}(s)=Y_{i 2}(s)+B_{i}(s) \text { for all } s .
\end{aligned}
$$

The first equation is for period 1 and says that income $Y_{i 1}$ equals consumption, $C_{i 1}$, plus the net investment in Arrow-Debreu (AD) assets: $p(s)$ is the market price for AD asset $s$ and $B_{i}(s)$ is the number of such assets bought by investor $i$. The second equation, says that for every state $s$, consumption equals period 2 income, $Y_{i 2}(s)$, plus the amount of AD asset $s$ bought in period 1 .

Investor $i$ 's first-order condition for $\mathrm{AD}$ asset $s$ is

$$
p(s)^{1 / \gamma} C_{i 2}(s)=\beta^{1 / \gamma} f_{i}(s)^{1 / \gamma} C_{i 1}
$$

Since this equation holds for every investor, it must also hold for a weighted average of them. Let $g_{i}$ be the shorthand notation for the cross-sectional pdf of different investors. Integrate the first-order conditions across investors to get

$$
p(s)^{1 / \gamma} \int_{i} C_{i 2}(s) g_{i} d i=\beta^{1 / \gamma} \int_{i} f_{i}(s)^{1 / \gamma} C_{i 1} g_{i} d i .
$$

Use the market clearing condition that output equals aggregate consumption in every state, $Y_{2}(s)=\int_{i} C_{i 2}(s) g_{i} d i$. For notational simplicity, let $Y_{2}(s)=s$ and solve for the price of 
asset $s$ as

$$
p(s)=\beta\left(\int_{i} f_{i}(s)^{1 / \gamma} C_{i 1} g_{i} d i\right)^{\gamma} / s^{\gamma} .
$$

This $\mathrm{AD}$ price is driven by how abundant the state is, the preference parameters ( $\beta$ and $\gamma$ ), and by a weighted average the beliefs (pdfs) of the different investors (raised to $1 / \gamma$ ) of the state. The weights in this average are $C_{i 1} g_{i}$, which can be thought of as the "economic weight" of investor $i$, since it is the product of his/her consumption and relative frequency (cross-sectional pdf). We clearly need the consumption choice in period $1\left(C_{i 1}\right)$ of every investor, to find the asset prices.

In general, there is no explicit solution for the consumption choice. However, in the special cases of logarithmic utility $(\gamma=1)$ or no disagreement, we know that all investors choose the same consumption in period 1 (if they have the same period 1 income), so the asset prices can readily be found. In other cases, we need to solve the model numerically or apply some sort of approximation. Once that is done, it is straightforward to derive other asset prices from the AD prices. ${ }^{2}$

This paper tries to analyse the effect of disagreement on asset prices in a very simple setting - to get clear results. Therefore, I assume that investors differ only with respect to their point forecasts of future output (income and preferences are the same across investors). In particular, investor $i$ thinks that $\log$ income (GDP), $\ln Y_{2}$, is normally distributed, that is,

$$
\text { investor } i \text { believes that } \ln Y_{2} \sim N\left(\mu_{i}, \sigma^{2}\right) \text {, }
$$

where $\mu_{i}$ is the point forecast (mean) of $\log$ output and $\sigma^{2}$ is the uncertainty. This means that $f_{i}(s)$ is a lognormal pdf.

I also assume that there is a continuum of investors who differ only with respect to their beliefs about the mean of $\ln Y_{2}, \mu_{i}$. In particular, I assume that the cross-sectional distribution of these means is also normal, that is,

$$
\text { the cross-section of investors is } \mu_{i} \sim N\left(\mu, \delta^{2}\right) \text {, }
$$

so $\delta^{2}$ is a measure of disagreement (cross-sectional variance) about future aggregate out-

\footnotetext{
${ }^{2}$ For instance, a real bond pays 1 unit in every state, so its price is just the integral of $p(s)$ - and the log real interest rate is the logarithm of 1/bond price. Alternatively, a claim on average consumption (a proxy for equity) pays $s$ in state $s$, so its price is the integral of $p(s) s$-and the log return is the logarithm of $s$ /price of the claim. Similarly, the price of a call option on average consumption with strike price $K$ is the integral (from $s=K$ to infinity) of $p(s)(s-K)$.
} 
put. This means that $g_{i}$ is a normal pdf. Figure 1.a illustrates the pdfs of three different investors: a pessimist (33rd percentile in the cross-sectional distribution), the median and an optimist (67th percentile). ${ }^{3}$
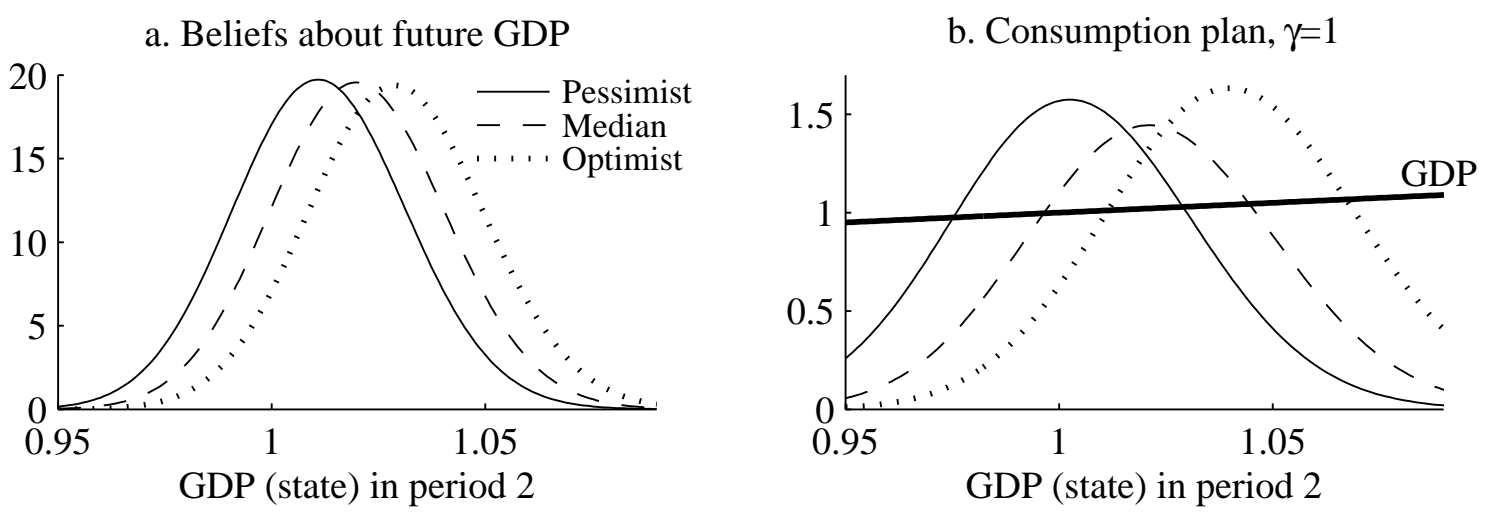

Figure 1: Beliefs and consumption plans for $\gamma=1$. This figure shows analytical results based on $\mu=\sigma=\delta=0.02$. The pessimist and optimist are the 33rd and 67th percentiles in the cross-sectional distribution.

\section{Asset Prices}

\subsection{Approximate Asset Prices}

It is possible to derive approximate analytical results for the asset prices if we assume that all investors have the same period 1 consumption. I will later show that this provides good approximations. With this assumption, the price of AD asset $s$ is (see Appendix B)

$$
p(s)=\left(\beta / s^{\gamma}\right)(\omega / \sigma)^{1-\gamma} \varphi\left(s ; \mu, \omega^{2}\right), \text { with } \omega^{2}=\sigma^{2}+\delta^{2} / \gamma,
$$

where first period income, $Y_{1}$, is normalised to unity and where $\varphi\left(s ; \mu, \omega^{2}\right)$ denotes the lognormal pdf with $\mathrm{E} \ln s=\mu$ and $\operatorname{Var}(\ln s)=\omega^{2}{ }^{4}$ The "variance" $\omega^{2}$ combines individual uncertainty and disagreement, but the latter is divided by the risk aversion $(\gamma)$. The AD prices are higher for states close to the mean and taper off in the tails. ${ }^{5}$

\footnotetext{
${ }^{3}$ Most models with heterogeneous beliefs have only two agents. Here, a continuum of different beliefs turns out to simplify the analysis (besides being more realistic).

${ }^{4}$ Clearly, the pdf is $\varphi\left(s ; \mu, \omega^{2}\right)=\exp \left[-(\ln s-\mu)^{2} /\left(2 \omega^{2}\right)\right] /(s \sqrt{2 \pi} \omega)$.

${ }^{5}$ Some general properties of the prices are as follows. First, states with high "probabilities" (pdf values) - according to most investors' beliefs_-are more expensive. Second, comparing two states with
} 
These approximate AD prices imply that the approximate $(\log )$ real interest rate $\left(r_{f}\right)$ and the cross-sectional (across investors) average expected excess return on a consumption claim $\left(\overline{\mathrm{E}} r_{c}-r_{f}\right)$ are

$$
\begin{aligned}
r_{f} & =-\ln \beta-(1-\gamma) \ln (\omega / \sigma)+\gamma \mu-\gamma^{2} \omega^{2} / 2, \\
\overline{\mathrm{E}} r_{c}-r_{f} & =(\gamma-1 / 2) \omega^{2}
\end{aligned}
$$

where $\omega^{2}$ is defined in (8). Given the AD prices, these expressions are exact so the approximation is due to the fact that the $\mathrm{AD}$ prices are approximate. (See Appendix $\mathrm{B}$ for derivations.) These are the standard expressions obtained from assuming normally distributed consumption growth (see, for instance, Campbell, Lo, and MacKinlay (1997), Ch. 8), except that $\omega^{2}$ is the relevant measure of "uncertainty." As usual, the consumption claim is used as a proxy for equity. The riskfree rate is increasing in disagreement if $\gamma>1$ (similar to Basak (2005)) and the same is true for the equity risk premium if $\gamma>1 / 2$. In addition, the equity risk premium is always increasing in the risk aversion coefficient.

The approximate AD prices also imply that the approximate option prices on the consumption claim follow the Black-Scholes formula, with "volatility" $\omega$. The basic reason is that future returns (consumption) are assumed to be lognormally distributed. In short,

$$
\text { option prices follow Black-Scholes with "volatility" } \omega=\sqrt{\sigma^{2}+\delta^{2} / \gamma} \text {. }
$$

As before, given the $\mathrm{AD}$ prices, this expressions is exact so the approximation is due to the fact that the AD prices are approximate. (See Appendix B for derivations.) This result generalises the results on implied volatilities in Shefrin (2005) Ch. 21 (which deals with the log utility case).

The basic message of this paper is that $\omega^{2}$ is (approximately) the relevant "uncertainty" for asset prices-and that disagreement is likely to play a relatively minor role when risk aversion is reasonably high. For instance, if individual uncertainty is as large as the disagreement $(\sigma=\delta)$ and the risk aversion $(\gamma)$ is three, then only a quarter of the equity premium in (10) is due to disagreement. With higher risk aversion, it is even less. This is illustrated in Figure 2.a which shows how the equity risk premium changes with the risk aversion $\gamma$-according to the approximation (10) for a case where disagreement

the same probability, the poorer state is more expensive. Third, a higher risk aversion $(\gamma)$ typically implies higher prices for poor states and lower prices for abundant states. 
is as large as the individual uncertainty $(\sigma=\delta=0.02)$ and for a case without disagreement $(\sigma=0.02, \delta=0)$. It is clear that disagreement accounts for a smaller fraction of the equity risk premium as risk aversion increases from $\gamma=1 / 2$ to $\gamma=6$.

a. Equity premium

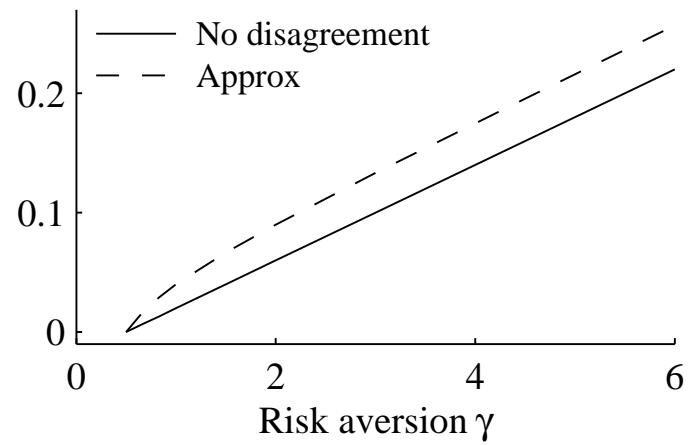

b. Pessimist's consumption plan

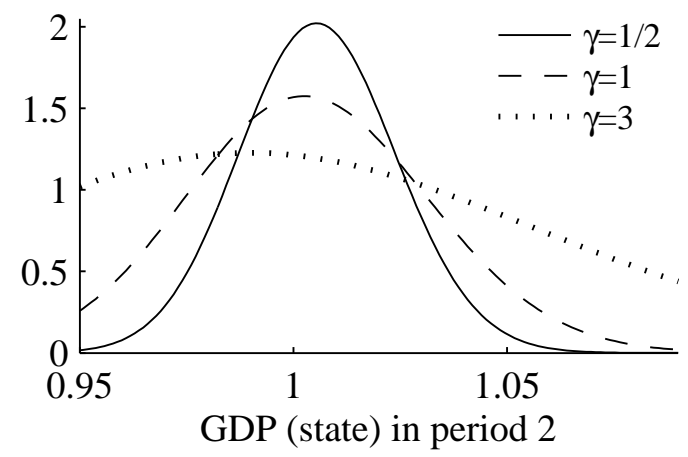

Figure 2: Equity risk premia consumption plan for different values of risk aversion $(\gamma)$. The baseline values are $\sigma=\delta=0.02$ and the case with no disagreement uses $\sigma=0.02, \delta=0$. See Appendix A for details on the numerical solution.

To explore this argument — and why disagreement matters at all for asset pricing — the next sections show exact solutions of the model for the two cases where that is possible (logarithmic utility or no disagreement) and numerical solutions for other cases.

\subsection{Exact Asset Prices (Logarithmic Utility or No Disagreement)}

The approximate asset prices and returns in (8)-(11) actually hold exactly when the utility function is logarithmic $(\gamma=1)$. The reason is that all investors then choose the same consumption level in period 1 (see Rubinstein (1974) and Detemple and Murthy (1994)). In this case, the relevant uncertainty of a "representative investor" is $\omega^{2}=\sigma^{2}+\delta^{2}$, so individual uncertainty $\left(\sigma^{2}\right)$ and disagreement $\left(\delta^{2}\right)$ are equally important.

With logarithmic utility, it is straightforward to derive the consumption plan for investor $i$ in each state, which helps understanding what disagreement implies for allocations and pricing. (See Appendix B for details.) Figure 1.b shows the consumption plans, $C_{i 2}(s)$, of three different investors (pessimist, median and optimist). Period 2 income, which would be the consumption for everyone if there were no disagreement, is also plotted. A consumption plan over (below) period 2 income in a state means that the investor buys (sells) the $\mathrm{AD}$ asset for that state. 
The figure illustrates that the main effect of disagreement is to make consumption more volatile than without disagreement (when all investors consume the common period 2 income): a pessimist $\left(\mu_{i}<\mu\right)$ tilts his portfolio towards assets that he thinks will pay off-and thus becomes less diversified. He thinks he gets good deals on those assets, since optimists are willing to supply them cheaply. The extra volatility induces a high risk premium on assets that are positively correlated with the investor's consumption. This is the basic mechanism for why disagreement matters for asset pricing. (David (2008) discusses this result in a more general model.)

The asset prices and returns in (8)-(11) also hold exactly when investors agree $(\delta=$ 0 - - since they then all make the same choices. In this case, the relevant uncertainty is $\omega^{2}=\sigma^{2}$. The approximate results can thus be seen as an approximation around either logarithmic utility or no disagreement.

\subsection{Numerically Calculated Asset Prices}

In this section I solve the model numerically by discretising the continuous state space (into 275 states) and the continuous cross-section of investors (into 51 investors). In the baseline case, I use the following parameter values: $\beta=1, \mu=\sigma=\delta=0.02$. This corresponds to an expected output growth of $2 \%$ with an individual standard deviation of $2 \%$ and also a cross-sectional standard deviation of $2 \%$. The individual uncertainty and the disagreement are thus the same. The volatility numbers are plausible for 3-year GDP growth according the survey evidence below. The time discount rate and the average growth rate do not matter for the risk premia. (See Appendix A for details.)

To get an intuition for why the effect of disagreement becomes smaller (in relative terms) as risk aversion increases, Figure 2.b shows numerical solutions of the consumption plan of a pessimist (33rd percentile in the cross-sectional distribution). The three different curves correspond to $\gamma=1 / 2,1$ and 3 . The overall pattern is the same as discussed in conjunction with logarithmic utility (see Figure 1.b): the pessimist concentrates his consumption to poor states, because he puts high probabilities on them. However, this behaviour is much less aggressive when the risk aversion is high. This effectively reduces the volatility of individual consumption, which counterbalances the higher risk premium that naturally follows from a higher risk aversion.

To assess the accuracy of the approximate asset prices and returns in (8)-(11), Figure 3 compares the numerical and approximate results on the equity premium and the implied 
volatility. Figure 3.a shows how the equity premium increases as the coefficient of relative risk aversion (horizontal axis) changes from $\gamma=1 / 2$ to $\gamma=6$. The two curves indicate the numerical (solid) and the approximate solution (dashed)—calculated using the baseline parameter values. The two solutions coincide at $\gamma=1$ (the "approximate" solution is then exact), but are very similar also for other values of the risk aversion.

Figure $3 . b$ is similar, except that disagreement is scaled up dramatically ( $\delta^{2}$ is five times higher than before). This figure is meant to illustrate very large disagreement (relative to individual uncertainty). For instance, the 33rd percentile investor (according to the cross-sectional distribution) only assigns a $2.5 \%$ probability to output growth higher than

a. Equity premium, baseline

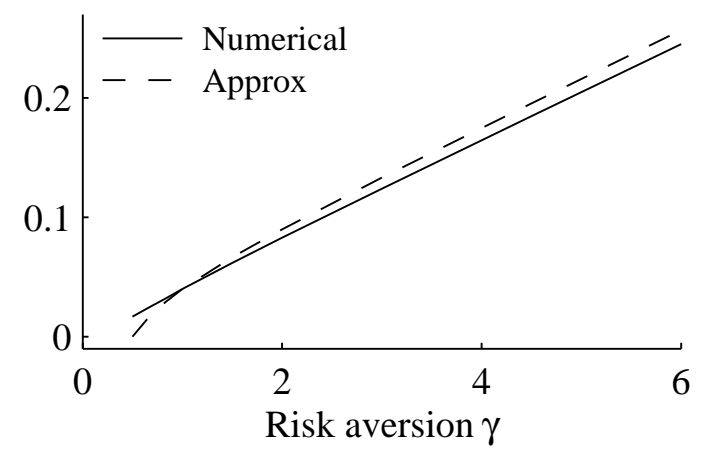

c. Implied volatility, baseline

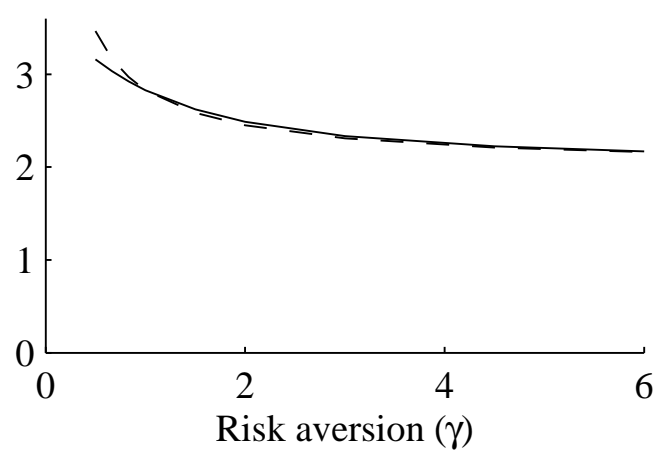

b. Equity premium, large disagreement

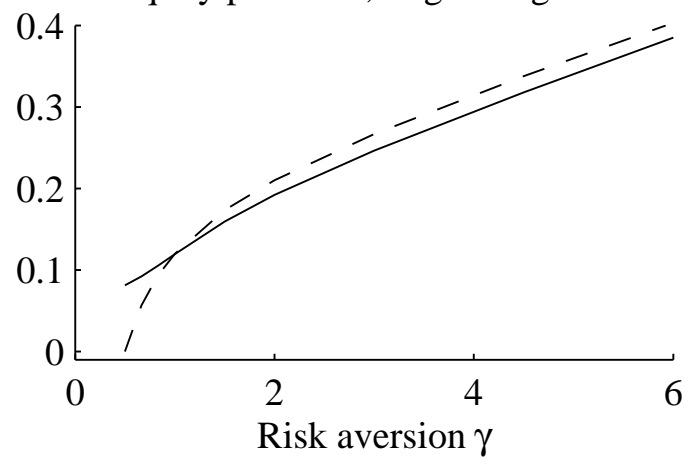

d. Implied volatility, large disagreement

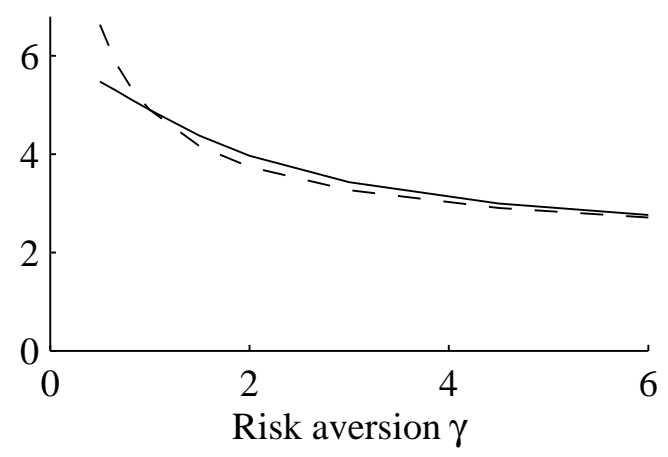

Figure 3: Equity risk premia and option implied volatilities for different values of risk aversion $(\gamma)$. The baseline values are $\sigma=\delta=0.02$. The case with large disagreement uses $\delta=0.045$. The implied volatility (standard deviation) from options is calculated at a strike price equal to the forward price of the consumption claim (the price of a consumption claim times $\exp \left(r_{f}\right)$ ). See Appendix A for details on the numerical solution. 
the point forecast made by the 67 th percentile investor. The effect of this is a considerable difference (in relative terms) between the numerical and approximate solutions for risk aversions below unity-but the fit is still good for risk aversions of one and above. In either case, the equity premium seems to be a monotonically increasing function of risk aversion (which is different from the result in David (2008)).

Figures 3.c-d repeat this exercise, but for the implied volatility (standard deviation) from call options-obtained by backing out the unknown standard deviation from the Black-Scholes formula. The calculations use options which are at the money, that is, when the strike price equals the forward price of the consumption claim. (Unreported results show that other strike prices give very similar results.) Once again, the numerical and approximate results are very similar, except for the combination of very low risk aversion and very large disagreement.

The main claim of this paper-that disagreement plays only a minor role if risk aversion is reasonably high-is clearly visible from both the numerical and approximate solutions (which are very similar in that case).

The reason for why the approximation works fine is that a high risk aversion implies a low elasticity of intertemporal substitution, so most investors choose fairly similar (expected) consumption in the two periods. With equal endowments this means that most investors choose fairly similar consumption levels in the initial period (which is the basic assumption in the approximation). ${ }^{6}$

\section{Survey Evidence on Individual Uncertainty and Disagreement}

The Survey of Professional Forecasters includes evidence on professional forecasters' beliefs about GDP. It asks for probability distributions (histograms) of GDP growth for the current and the next calendar years, and also about the point forecasts (no histograms) about the average growth over the next 10 years (see Croushore (1993) for details). A particular strength of this data is that contains information about both disagreement and individual uncertainty - in contrast to most surveys (for instance, the data on analysts used in Anderson, Ghysel, and Juergens (2005)).

\footnotetext{
${ }^{6}$ With low risk aversion, this is no longer true since some investors are happy to save a bit more today in order to exploit (what they think are) great deals on assets that pay off in future states they put high probabilities on. This makes period 1 consumption more diverse, which causes a larger approximation error.
} 
I fit distributions to the individual histograms as follows. In the uncommon case where only one bin is used (that is, the respondent puts $100 \%$ of the probability on one bin), I assume a triangular distribution within that bin. If two bins or more are used, then a normal distribution is fitted (a mean and a variance). The fitting is done by minimising the sum of the squared deviations of the theoretical from the observed probabilities (see Giordani and Söderlind (2003)).

This estimation gives a cross-section of individual point forecasts (means) and variances in each time period (the first quarter of each year 1992-2007). Since there are occasional strange survey answers (typos?), I apply robust estimators to the cross-section in each period: "average" individual uncertainty is estimated by the median variance (from the histograms) and disagreement is estimated by the square of the interquartile range (divided by $1.35^{2}$ to be comparable to a variance).

Table 1 shows time-averages of these results. Individual uncertainty is more than twice as large as the disagreement for both the first and the second year. In addition, the disagreement about average 10-year growth is just half of the disagreement about 1 -year growth. This suggests that it is plausible to assume that individual uncertainty dominates disagreement also for longer horizons (unfortunately, there is no data on 10year individual uncertainty).

\begin{tabular}{lrr} 
& $\begin{array}{r}\text { Median individual } \\
\text { variance, } \sigma^{2}\end{array}$ & $\begin{array}{r}\text { Cross-sectional } \\
\text { variance, } \delta^{2}\end{array}$ \\
\hline Current year & 0.41 & 0.19 \\
Next year & 0.62 & 0.24 \\
Next 10 years (average) & & 0.11 \\
\hline
\end{tabular}

Table 1: Individual uncertainty and disagreement about GDP growth, Survey of Professional Forecasters. The table shows time-averages from Q1 surveys 1992-2007 by the Survey of Professional Forecasters. The median individual variance (for a given period) is estimated from the fitted individual variances. The cross-sectional variance (for a given period) is estimated by the square of the inter-quartile range of the individual point estimates, divided by $1.35^{2}$. The latter is a robust estimator of the variance and coincides with a traditional estimator in case the data is normally distributed. 


\section{Discussion}

This paper shows two things. First, analytical solutions of a two-period endowment model suggest that the uncertainty of a "representative investor" is approximately $\sigma^{2}+\delta^{2} / \gamma$, where $\sigma^{2}$ denotes individual uncertainty, $\delta^{2}$ is a measure of disagreement (cross-sectional variance of investors' point estimates) and $\gamma$ is the coefficient of relative risk aversion. Comparison with numerical solutions suggest that this is a good approximation-if the relative risk aversion is one or higher. Second, empirical results from the survey data from the Survey of Professional Forecasters indicate that individual uncertainty is actually larger than disagreement.

If we are willing to believe in a high relative risk aversion, then the two results together suggest that disagreement plays only a small role in asset pricing.

There are, of course, many caveats. In particular, the model is very simple and therefore leaves out potentially important aspects. It might also be the case that disagreement is more important on financial markets than what the empirical evidence from the Survey of Professional Forecasters show. Finally, we cannot be sure that the relevant degree of risk aversion is high. Evidence from surveys and experiments is still mixed (see, for instance Barsky, Juster, Kimball, and Shapiro (1997) and Holt and Laury (2002)), although empirical studies of consumption-based models (with or without refinements like habit persistence) suggests high numbers (see, for instance, Campbell (2003)).

Still, the paper demonstrates that basic asset pricing theory does not necessarily assign an important role to disagreement.

\section{A Numerical Solution of the Model}

This appendix summarises the numerical solution of the model.

The (continuous) cross-section of investors is approximated by $m$ investors: the means $\left(\mu_{i}, i=1, \ldots, m\right)$ are the $i /(m+1)$ percentiles of the $N\left(\mu, \delta^{2}\right)$ distribution. The (continuous) state space is approximated by $n$ states, equally spaced (in logs) from percentile $\theta$ of the most pessimistic investor to percentile $1-\theta$ of the most optimistic investor. The probability of state $s$ for investor $i$ is calculated as the integral of a $N\left(\mu_{i}, \sigma^{2}\right)$ pdf (denoted $\left.f_{i}(s)\right)$ over the interval $(s-\lambda, s+\lambda)$, where $\lambda$ is halfway to the next state. 
The results are obtained by solving the following system of equations (in terms of $C_{i 1}$ )

$$
\begin{aligned}
Y_{i 1}-C_{i 1}-\sum_{s=1}^{m} p(s) B_{i}(s)=0 \text { for } i=1, \ldots, m, \text { where } \\
p(s)=\left(\sum_{i=1}^{m} f_{i}(s)^{1 / \gamma} C_{i 1} / m\right)^{\gamma} / s^{\gamma} \text { for } s=1, \ldots, n, \\
C_{i 2}(s)=f_{i}(s)^{1 / \gamma} p(s)^{-1 / \gamma} C_{i 1}, \\
B_{i}(s)=C_{i 2}(s)-Y_{i 2}(s) .
\end{aligned}
$$

In the calculations $n=275, m=51$ and $\theta=1 e-6$.

\section{B Derivations of Asset Prices}

When $C_{i 1}$ is the same for all investors, (or more generally, when $f_{i}(s)^{1 / \gamma}$ and $C_{i 1}$ are uncorrelated), then (5) can be written

$$
p(s)=\beta\left(\int_{i} C_{i 1} g_{i} d i\right)^{\gamma}\left(\int_{i} f_{i}(s)^{1 / \gamma} g_{i} d i\right)^{\gamma} / s^{\gamma}=\beta\left(\int_{i} f_{i}(s)^{1 / \gamma} g_{i} d i\right)^{\gamma} / s^{\gamma},
$$

since $\int_{i} C_{i 1} g_{i} d i$ equals average income in period 1 , which is normalised to unity. Straightforward integration gives (8). (See also Giordani and Söderlind (2005) for details on the integration.)

Using the approximate asset prices (8) and integrating, $p_{r}=\int_{s} p(s) d s$ and $p_{c}=$ $\int_{s} p(s) s d s$ gives

$$
\begin{aligned}
& p_{r}=\beta(\omega / \sigma)^{1-\gamma} \exp \left(-\gamma \mu+\gamma^{2} \omega^{2} / 2\right), \\
& p_{c}=\beta(\omega / \sigma)^{1-\gamma} \exp \left((1-\gamma) \mu+(1-\gamma)^{2} \omega^{2} / 2\right) .
\end{aligned}
$$

The $\log$ riskfree rate, $\ln \left(1 / p_{r}\right)$, is then as in (9) and the excess $\log$ return on the consumption claim, $\ln \left(Y_{2} / p_{c}\right)-\ln \left(1 / p_{r}\right)$, is

$$
r_{c}-r_{f}=-\mu-(1-2 \gamma) \omega^{2} / 2+\ln Y_{2}
$$

Take the average expectations over $\ln Y_{2}, \int_{i} \mu_{i} g_{i} d i=\mu$, to get (10).

To price a call option on the consumption claim with strike price $K$, use the approxi- 
mate asset prices (8) and integrate, $\int_{s=K}^{\infty} p(s)(s-K) d s$ to get

$$
\begin{aligned}
& (\omega / \sigma)^{1-\gamma} \exp \left((1-\gamma) \mu+(1-\gamma)^{2} \omega^{2} / 2\right) \frac{1}{2} \operatorname{erfc}\left(\frac{(\gamma-1) \omega^{2}-\mu+\ln K}{\sqrt{2} \omega}\right)- \\
& (\omega / \sigma)^{1-\gamma} \exp \left(-\gamma \mu+\gamma^{2} \omega^{2} / 2\right) K \frac{1}{2} \operatorname{erfc}\left(\frac{\gamma \omega^{2}-\mu+\ln K}{\sqrt{2} \omega}\right),
\end{aligned}
$$

where erfc is the complementary error function. This can be simplified by noticing a few things. First, the exponential part of the first term equals $p_{c}$ and the exponential part of the second term equals $p_{r}$ (see above). Second, $\operatorname{erfc}(b / \sqrt{2}) / 2=\Phi(-b)$, where $\Phi()$ is the cumulative distribution function of a standard normal distribution. Third, the negative of the argument of the first erfc function can be rewritten as $\ln \left(p_{c} / p_{r}\right)+\omega^{2} / 2$ (use the definitions of $p_{c}$ and $p_{r}$ from above). Fourth, the negative of the argument of the second erfc function equals the negative of the argument of the first erfc function minus $\omega$. Together, these simplifications give the call option price

$$
p_{c} \Phi\left(d_{1}\right)-p_{r} K \Phi\left(d_{1}-\omega\right), \text { where } d_{1}=\frac{\ln p_{c} / K-\ln p_{r}+\omega^{2} / 2}{\omega},
$$

which is the Black-Scholes formula (recalling that $p_{r}=\exp \left(-r_{f}\right)$ ), where $\omega^{2}$ plays the role of the volatility (variance).

The formulas for the approximate asset prices apply also to the cases of logarithmic utility $(\gamma=1)$ and no disagreement $(\delta=0$, so $\omega=\sigma)$-but then exactly (since $C_{i 1}$ is indeed the same for all investors).

With logarithmic utility, the asset prices are

$$
p(s)=(\beta / s) \varphi\left(s ; \mu, \sigma^{2}+\delta^{2}\right)
$$

Combine with (3) (with $\gamma=1$ ) and $f_{i}(s)=\varphi\left(s ; \mu_{i}, \sigma^{2}\right)$ as well as $C_{i 1}=1$ to get period 2 consumption

$$
C_{i 2}(s)=s \frac{\varphi\left(s ; \mu_{i}, \sigma^{2}\right)}{\varphi\left(s ; \mu, \sigma^{2}+\delta^{2}\right)} .
$$

With no disagreement, the asset prices are

$$
p(s)=\left(\beta / s^{\gamma}\right) \varphi\left(s ; \mu, \sigma^{2}\right) .
$$




\section{References}

Anderson, E. W., E. Ghysel, and J. L. Juergens, 2005, "Do heterogenous beliefs matter for asset pricing," Review of Financial Studies, 18, 875-924.

Barsky, R. B., F. T. Juster, M. S. Kimball, and M. D. Shapiro, 1997, "Preference parameters and behavioral heterogeneity: an experimental approach in the health and retirement study," The Quarterly Journal of Economics, 112, 537-579.

Basak, S., 2005, "Asset pricing with heterogenous agents," Journal of Banking and Finance, 29, 2849-2881.

Campbell, J. Y., 2003, “Consumption-based asset pricing," in George Constantinides, Milton Harris, and Rene Stultz (ed.), Handbook of the Economics of Finance . chap. 13, North-Holland, Amsterdam.

Campbell, J. Y., A. W. Lo, and A. C. MacKinlay, 1997, The econometrics of financial markets, Princeton University Press, Princeton, New Jersey.

Croushore, D., 1993, "Introducing: the Survey of Professional Forecasters," Federal Reserve Bank of Philadelphia Business Review, pp. 3-13.

David, A., 2008, "Heterogeneous beliefs, speculation, and the equity premium," Journal of Finance, Volume 63, 41-83.

Detemple, J., and S. Murthy, 1994, "Intertemporal asset pricing with heterogeneous beliefs," Journal of Economic Theory, 62, 294-320.

Fama, E. F., and K. R. French, 2007, "Disagreement, tastes, and asset prices," Journal of Financial Economics, 83, 667-689.

Gerber, A., and T. Hens, 2006, "Modelling alpha-opportunities within the CAPM," Working Paper 317, NCCR FINRISK.

Giordani, P., and P. Söderlind, 2003, "Inflation forecast uncertainty," European Economic Review, 47, 1037-1059. 
Giordani, P., and P. Söderlind, 2005, "Is there evidence of pessimism and doubt in subjective distributions? Implications for the equity premium puzzle," Journal of Economic Dynamics and Control, 30, 1027-1043.

Holt, G. A., and S. K. Laury, 2002, "Risk aversion and incentive effects," The American Economic Review, 92, 1644-1655.

Hong, H., and J. C. Stein, 2007, "Disagreement and the stock market," Journal of Economic Perspectives, 21, 109-128.

Kurz, M., and M. Motolese, 2007, "Diverse beliefs and time variability of risk premia," mimeo, Stanford University.

Lintner, J., 1969, “The aggregation of investor's diverse judgments and preferences in purely competitive security markets," The Journal of Financial and Quantitative Analysis, 4, 347-400.

Rubinstein, M., 1974, “An aggregation theorem for securities markets," Journal of Financial Economics, 1, 225-244.

Shefrin, H., 2005, A behavioral approach to asset pricing, Elsevier Academic Press.

Varian, H. R., 1985, “Divergence of opinion in complete markets: a note," Journal of Finance, 40, 309-317. 